\title{
Measuring the Virial Temperature of Galactic Halos through Electron Scatteringof Quasar Emission Lines
}

\section{Citation}

Loeb, Abraham. 1998. "Measuring the Virial Temperature of Galactic Halos through Electron Scatteringof Quasar Emission Lines." The Astrophysical Journal 508 (2): L115-18. https:// doi.org/10.1086/311744.

\section{Permanent link}

http://nrs.harvard.edu/urn-3:HUL.InstRepos:41393222

\section{Terms of Use}

This article was downloaded from Harvard University's DASH repository, and is made available under the terms and conditions applicable to Other Posted Material, as set forth at http:// nrs.harvard.edu/urn-3:HUL.InstRepos:dash.current.terms-of-use\#LAA

\section{Share Your Story}

The Harvard community has made this article openly available. Please share how this access benefits you. Submit a story. 


\title{
MEASURING THE VIRIAL TEMPERATURE OF GALACTIC HALOS THROUGH ELECTRON SCATTERING OF QUASAR EMISSION LINES
}

\author{
ABRAHAM LOEB \\ Astronomy Department, Harvard University, 60 Garden Street, Cambridge, MA 02138; aloeb@cfa.harvard.edu \\ Received 1998 September 8; accepted 1998 October 6; published 1998 October 23
}

\begin{abstract}
Semianalytic models of galaxy formation postulate the existence of virialized gaseous halos around galaxies at high redshifts. A small fraction of the light emitted by any high-redshift quasar is therefore expected to scatter off the free electrons in the halo of its host galaxy. The broadening of the scattered emission lines of the quasar can be used to measure the temperature of these electrons. For gas in virial equilibrium, the velocity width of the scattered line profile is larger by a factor of $\sim\left(m_{p} / m_{e}\right)^{1 / 2}=43$ than the velocity dispersion of the host galaxy and reaches $\gtrsim 10,000 \mathrm{~km} \mathrm{~s}^{-1}$ for the massive galaxies and groups in which bright quasars reside. In these systems, the scattered width exceeds the intrinsic width of the quasar lines and hence can be used to measure the virial temperature of the quasar host. The high degree of polarization of the scattered radiation can help filter out the extended scattered light from the central emission by the quasar and its host galaxy. The signal-to-noise ratio of the spectral broadening can be enhanced by matching the full spectrum of the scattered radiation to a template of the unscattered quasar spectrum. Although the central fuzz around low-redshift quasars is dominated by starlight, the fuzz around high-redshift quasars might be dominated by scattering before galaxies have converted most of their gas reservoirs into stars.
\end{abstract}

Subject headings: galaxies: fundamental parameters — quasars: emission lines

\section{INTRODUCTION}

The depth of galactic potential wells has been traditionally inferred by dynamical methods (H I or stellar rotation curves and satellite dynamics) or gravitational lensing. Unfortunately, the application of these methods to galaxies at very high redshifts proves difficult. The resulting uncertainty in the velocity dispersion of galactic halos prevents current data sets on the abundance and clustering of galaxies at $z \sim 3$ from being able to discriminate among different cosmological models (Adelberger et al. 1998).

Semianalytic models of galaxy formation and evolution (e.g., Kauffmann, White, \& Guiderdoni 1993; Baugh et al. 1998; Somerville, Primack, \& Faber 1998) postulate the existence of gaseous halos at the virial temperature around galaxies at high redshifts. The halos are expected to contain gas that was assembled into the galaxies but was unable to cool between successive heating episodes, caused either by merger events or by supernova-driven winds. The supply of cold gas from the dense core of these halos controls the star formation rate of their host galaxies. Although X-ray halos are rare among local galaxies (Bregman \& Glassgold 1982; Fabbiano 1989), they might have been prominent during their early evolution. A direct measurement of the gas temperature in these halos could determine the potential depth of their host galaxy.

In this Letter, we propose a novel method for probing the virial temperature of galactic halos in those cases in which a bright quasar shines at their center. The quasar radiation is scattered by the free electrons in the halo, and the scattered emission lines are Doppler broadened by the thermal velocity of these electrons. ${ }^{1}$ Due to the small electron mass, the velocity width of the scattered lines is larger by a factor of $\sim\left(m_{p} / m_{e}\right)^{1 / 2}=43$ than the velocity dispersion of the galaxy and could reach values of $\gtrsim 10,000 \mathrm{~km} \mathrm{~s}^{-1}$ for the massive

\footnotetext{
${ }^{1}$ Interestingly, this broadening effect was only recently detected in a local astrophysical setting - the solar wind-by the $\mathrm{SOHO}$ satellite (Fineschi et al. 1998).
}

galaxies and groups in which bright quasars reside. In these objects, the scattered line width exceeds the typical intrinsic width of the quasar emission lines (FWHM $\sim 5000 \mathrm{~km} \mathrm{~s}^{-1}$; see, e.g., Peterson 1997 for a review) and can be used to measure the electron temperature. Since quasars tend to reside in dense environments such as groups of galaxies (Hartwick \& Schade 1990; Bahcall \& Chokshi 1991; Ellingson, Green, \& Yee 1991; Fisher et al. 1996), additional scattering may also result from the intragroup gas.

In $\S 2$ we calculate the expected shape and amplitude of the scattered quasar lines as a function of the gas temperature and density profile. Section 3 discusses the implications of our results.

\section{SCATTERED LINE PROFILE}

The redistribution function $R\left(\nu^{\prime}, \boldsymbol{n}^{\prime} ; \nu, \boldsymbol{n}\right) d \nu^{\prime} d \nu\left(d \Omega^{\prime} / 4 \pi\right) \times$ $(d \Omega / 4 \pi)$ provides the probability of scattering a photon from a frequency between $\left(\nu^{\prime}, \nu^{\prime}+d \nu^{\prime}\right)$ and solid angle $d \Omega^{\prime}$ in the direction $\boldsymbol{n}^{\prime}$ to a frequency between $(\nu, \nu+d \nu)$ and solid angle $d \Omega$ in the direction $\boldsymbol{n}$. For Thomson scattering by a Maxwellian population of electrons at a temperature $T$, the redistribution function is given by (Dirac 1925; Mihalas 1978)

$$
\begin{aligned}
R\left(\nu^{\prime}, \boldsymbol{n}^{\prime} ; \nu, \boldsymbol{n}\right)=\frac{3}{4}\left(1+\mu^{2}\right) & \frac{1}{\left[2 \pi \beta_{T}^{2}(1-\mu) \nu^{2}\right]^{1 / 2}} \\
& \times \exp \left[-\frac{\left(\nu-\nu^{\prime}\right)^{2}}{2 \beta_{T}^{2}(1-\mu) \nu^{2}}\right],
\end{aligned}
$$

where $\mu=\boldsymbol{n}^{\prime} \cdot \boldsymbol{n}=\cos \theta$, and

$$
\beta_{T} \equiv\left(\frac{2 k T}{m_{e} c^{2}}\right)^{1 / 2}
$$

is the typical thermal velocity of an electron in units of the speed of light $c$. Here $m_{e}$ is the electron mass, and $k$ is Boltz- 
mann's constant. The redistribution function can be integrated over any arbitrary scattering geometry to obtain the profile of a scattered quasar line.

As an illustrative example, we consider below the case of a spherical, optically thin gas cloud that scatters a narrow line of frequency $\nu_{0}$ and total luminosity $L_{0}$ from a central source. We assume that the source luminosity per unit frequency is $L_{\nu^{\prime}}=L_{0} \delta\left(\nu^{\prime}-\nu_{0}\right)$. An electron at a radius $r$ scatters a net fraction $\left(\sigma_{\mathrm{T}} / 4 \pi r^{2}\right)$ of $L_{0}$, where $\sigma_{\mathrm{T}}=0.67 \times 10^{-24} \mathrm{~cm}^{2}$ is the Thomson cross-section. Hence, the scattered spectral luminosity at a frequency $\nu$ is

$$
\begin{aligned}
L_{\nu}= & \int d^{3} r n_{e}\left(\frac{\sigma_{\mathrm{T}}}{4 \pi r^{2}}\right) \int \frac{d \Omega}{4 \pi} \int \frac{d \Omega^{\prime}}{4 \pi} \\
& \times \int d \nu^{\prime} R\left(\nu^{\prime}, \boldsymbol{n}^{\prime} ; \nu, \boldsymbol{n}\right) L_{\nu^{\prime}}\left(\nu^{\prime}\right),
\end{aligned}
$$

where $n_{e}(r)$ is the number density of electrons at a radius $r$. By substituting $d^{3} r=4 \pi r^{2} d r$ and $L_{\nu^{\prime}}=L_{0} \delta\left(\nu^{\prime}-\nu_{0}\right)$ and integrating by parts, we get (Rybicki \& Hummer 1994)

$$
\begin{aligned}
\frac{\beta_{T} \nu_{0} L_{\nu}}{\tau L_{0}}= & \beta_{T} \nu_{0} \int_{-1}^{1} \frac{d \mu}{2} R\left(\nu_{0}, \nu, \mu\right) \\
= & {\left[\left(\frac{11}{10}+\frac{2}{5} \Delta^{2}+\frac{\Delta^{4}}{20}\right) \frac{e^{-\Delta^{2 / 4}}}{\sqrt{\pi}}\right.} \\
& \left.-\left(\frac{3}{2}+\frac{\Delta^{2}}{2}+\frac{\Delta^{4}}{20}\right) \frac{|\Delta|}{2} \operatorname{erfc}\left(\frac{|\Delta|}{2}\right)\right],
\end{aligned}
$$

where $\operatorname{erfc}(x) \equiv(2 / \sqrt{\pi}) \int_{x}^{\infty} \exp \left(-t^{2}\right) d t$ is the complementary error function,

$$
\Delta \equiv \frac{\left(\nu-\nu_{0}\right)}{\beta_{T} \nu_{0}}
$$

is the frequency shift relative to the line center in units of the typical Doppler shift of a thermal electron, and

$$
\tau=\int_{0}^{\infty} d r n_{e} \sigma_{\mathrm{T}}
$$

is the radial optical depth of the halo. The integral of the righthand side of equation (4) over $\Delta$ is unity. The scattered profile of a line with a finite initial width can be obtained by convolving the initial line profile with the kernel given by equation (4). This kernel is plotted as the solid line in Figure 1.

The effective width of the scattered line can be found from the second moment, $\left\langle\Delta^{2}\right\rangle=\left(\int \Delta^{2} L_{\nu} d \Delta\right) / \int L_{\nu} d \Delta$. We find

$$
\frac{\delta \nu}{\nu} \equiv\left\langle\left(\frac{\nu-\nu_{0}}{\nu_{0}}\right)^{2}\right\rangle^{1 / 2}=\beta_{T}
$$

where the angular brackets denote an average over $L_{\nu}$.

Next, we apply the above results to the particular halo profile of an isothermal sphere with a one-dimensional velocity dispersion $\sigma_{v}$. For a fully ionized gas of electrons and protons, $k T=m_{p} \sigma_{v}^{2}$, where $m_{p}$ is the proton mass. We then get from

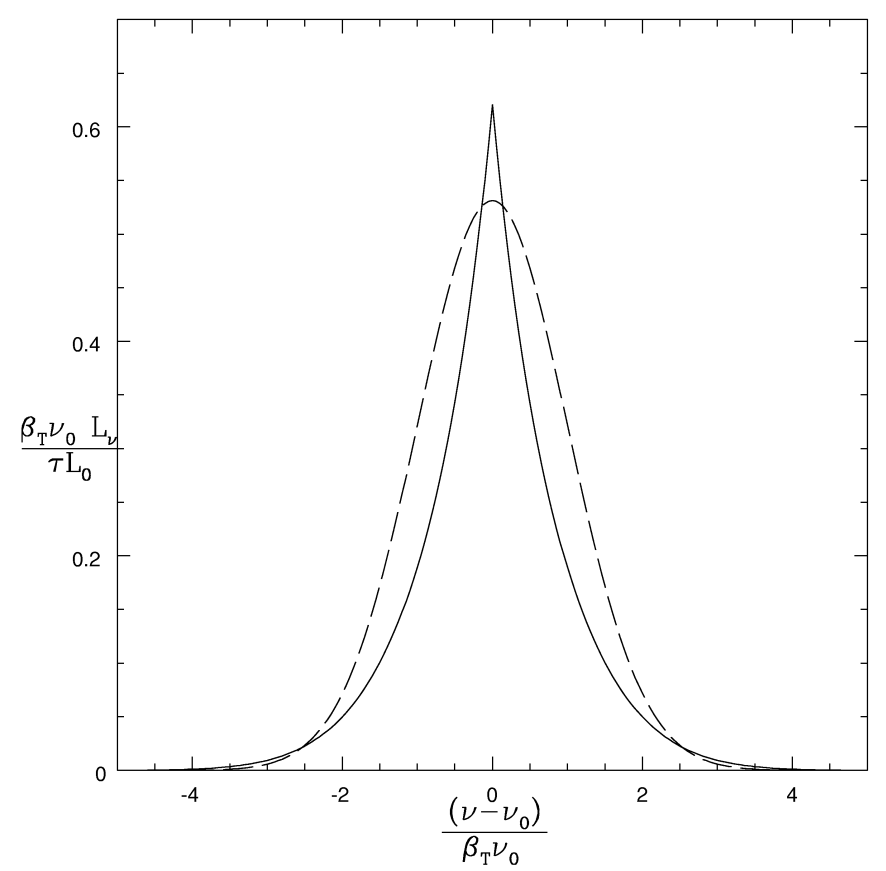

FIG. 1.-Profile of a scattered line from a spherical halo (solid line; see eq. [4]) in comparison with the profile for $90^{\circ}$ scattering and the same flux normalization (dashed line). Both profiles have a unit integral and a unit second moment.

equation (7)

$$
\frac{\delta \nu}{\nu} c=1.2 \times 10^{4} \sigma_{200} \mathrm{~km} \mathrm{~s}^{-1}
$$

where $\sigma_{200}=\left(\sigma_{v} / 200 \mathrm{~km} \mathrm{~s}^{-1}\right)$. We assume that the gas provides a fraction $f_{\text {gas }}$ of the total mass and follows the radial profile of the dark matter at large radii but has a core of radius $r_{c}$. Hence, we take $n_{e}=f_{\text {gas }} \sigma^{2} /\left[2 \pi G m_{p}\left(r_{c}^{2}+r^{2}\right)\right]$, where $G$ is Newton's constant. Equation (6) then yields the net fraction of the quasar light that gets scattered:

$$
\tau=2 \times 10^{-2} f_{10} \sigma_{200}^{2} r_{1}^{-1},
$$

where $f_{10} \equiv\left(f_{\text {gas }} / 10 \%\right)$ and $r_{1} \equiv\left(r_{\mathrm{c}} / 1 \mathrm{kpc}\right)$. The value of $f_{\text {gas }}$ can be as high as $20 \%$ if the cosmological density parameters of baryons and dark matter are 0.06 and 0.3 , respectively. The radius inside which the cooling time due to free-free emission is shorter than a fraction $\eta$ of the Hubble time is $\sim 25 \mathrm{kpc}\left(\eta f_{10} \sigma_{200}\right)^{1 / 2}[(1+z) / 4]^{-3 / 4}$. The relevant numerical value of $\eta \lesssim 1$ depends on the average fraction of the Hubble time that passes between major mergers, since these mergers could reheat the diffuse gas to its virial temperature. In fact, if quasar activity is triggered by galaxy mergers, then $\eta$ may be as small as the ratio between the dynamical timescale of the host galaxy (during which the quasar is fueled) and the Hubble time, namely $\sim 0.1(r / 30 \mathrm{kpc}) \sigma_{200}^{-1}[(1+z) / 4]^{3 / 2}$. Inside the cooling radius, hot gas may still be replenished by supernova-driven winds (in which case the geometry of the scattering material is likely to be nonspherical). The quasar radiation itself is expected to ionize and heat much of the quiescent interstellar medium as well, and so overall it is natural to expect $r_{1} \lesssim 10$. In resolution-limited observations for which the quasar has to be subtracted from the image, the effective value of $\tau$ would be dictated by the extent of the quasar mask, which sets 
a minimum value to $r$. Scattering by dust would also contribute to the diffuse light, but it would not broaden the quasar lines significantly. For the massive galaxies and groups in which bright quasars tend to reside (Hartwick \& Schade 1990; Bahcall \& Chokshi 1991; Ellingson et al. 1991; Fisher et al. 1996), the width of the scattered emission lines will typically be $\gtrsim 10^{4}$ $\mathrm{km} \mathrm{s}^{-1}$, i.e., in excess of their intrinsic width.

Note that the optical depth of a cluster of galaxies with $\sigma_{v} \sim 10^{3} \mathrm{~km} \mathrm{~s}^{-1}$ and a core radius $r_{c} \sim 250 \mathrm{kpc}$ is the same as that of a galaxy with $\sigma_{v} \sim 200 \mathrm{~km} \mathrm{~s}^{-1}$ and $r_{c} \sim 10 \mathrm{kpc}$. However, in the cluster case, the surface brightness of the scattered light is a factor of $25^{2}=625$ smaller and is much more difficult to separate from contaminating background light. Moreover, the lines scattered by the cluster would only appear as a broad hump on top of the continuum because of their large width, $(\delta \nu / \nu) \sim 0.2$. Scattering of beamed quasar light by intracluster gas was suggested as the origin of the observed alignment between the optical continuum and radio axes of high-redshift radio galaxies (Fabian 1989).

The signal-to-noise ratio for the detection of electron broadening can be improved by using a full template of the quasar spectrum with all of its lines, such as O VI, Ly $\alpha, \mathrm{C}$ IV, Mg II, and $\mathrm{H} \alpha, \mathrm{H} \beta$, and $\mathrm{H} \gamma$. The scattered lines will be centered on the template wavelengths and will be broadened by the same amount (except for the blue wing of the Ly $\alpha$ line, which gets absorbed by the Ly $\alpha$ forest of the intergalactic medium). The broadening due to electron scattering can be distinguished from the intrinsic width of the lines since it should be the same for all lines, while the intrinsic width is often different for different lines (Peterson 1997).

For a density profile $n_{e} \propto r^{-\alpha}$, the degree of polarization near the limb of the scattering halo is $P=(\alpha+1) /(\alpha+3)$ (Cassinelli \& Hummer 1971). In the singular isothermal sphere case, $\alpha=2$, and so $P=0.6$. Since the intrinsic quasar emission is often weakly polarized, this high degree of polarization can be used to filter out the scattered component from the emission component in the spectrum of the quasar and its host (and may also be used to reduce the surface brightness contrast between the quasar pointlike emission and the extended scattered light). Since the net polarization of a spherical halo is zero, the detection of polarization requires that the halo be resolved. At any point, the $\boldsymbol{E}$-vector polarization axis is perpendicular to the radius vector from the halo center.

In spectroscopic observations for which the halo is resolved, the line profile might deviate from the shape derived above. In particular, the spectral shape in equation (4) holds only if the flux is summed over a symmetric ring or over half of the symmetric projection of the entire halo on the sky (with the value of $\tau$ depending on geometry). If a spectrum is taken for any other portion of the halo, then the line profile would be different. For example, in the limit of high angular resolution (needed for the detection of polarization) and a steep electron density profile (for which the polarization approaches $100 \%$ ), the line shape would approach $R\left(\nu_{0}, \nu\right)$ for a scattering angle of $\theta=90^{\circ}(\mu=0)$, since most of the scattered flux would be contributed from the point of closest approach of the line of sight relative to the source. The dashed line in Figure 1 compares the $90^{\circ}$ scattering profile of equation (1) to the spectral shape given by equation (4).

\section{DISCUSSION}

We have shown that the scattered light of a central quasar can serve as a thermometer of hot galactic halos with a virial temperature $T=5 \times 10^{6} \sigma_{200}^{2} \mathrm{~K}$. This probe complements other methods for measuring the depth of galactic potential wells, although it is limited only to galaxies that harbor a quasar. ${ }^{2}$ Local observations of galactic centers (Magorrian et al. 1998) and popular models of quasar evolution (e.g., Haehnelt \& Rees 1993; Haiman \& Loeb 1998) suggest that all galaxies went through a quasar phase during their history, and so this method could be applied to a fair sample of all galaxies.

The scattered flux is expected to replicate the quasar spectrum with broadened line widths of $\sim 10^{4} \sigma_{200} \mathrm{~km} \mathrm{~s}^{-1}$ and to include up to a few percent of the quasar flux (see eqs. [8] and [9]). Its contrast relative to stellar emission by the host galaxy should increase toward the rest-frame UV. Current photometric observations of quasar hosts are sensitive to fuzz within several arcseconds $(\$ 10 \mathrm{kpc}$ ) from the quasar at a minimum level of $\sim 1 \%$ of the quasar emission (e.g., McLeod \& Rieke 1995; Bahcall et al. 1997, and references therein). The method described here requires low-resolution spectroscopy at a comparable contrast level. The signal-to-noise ratio of the spectroscopy can be improved considerably by filtering out only polarized light from a resolved halo image. Under more favorable contrast ratios, the feasibility of detecting extended Ly $\alpha$ emission around high-redshift quasars has already been demonstrated in many cases (e.g., Heckman et al. 1991a, 1991b; Bremer et al. 1992; Hu, McMahon, \& Egami 1996).

The scattering in elliptical galaxies might benefit also from the presence of a surrounding intragroup or intracluster gas. In spiral galaxies, the scattering by hot supernova-heated gas would add to the more diffuse scattering in the surrounding halo. The effect of hot interstellar gas in galactic disks would be heavily contaminated by dust scattering and absorption and by stellar emission. Although the scattering by dust should also be polarized, it would not increase significantly the width of the quasar emission lines. For example, the broad Mg II emission observed from the extended image of the radio galaxy 3C 265 (Dey \& Spinrad 1996) is most likely associated with dust scattering of nuclear emission based on its flux, polarization, and the constancy of the measured line width as a function of radius.

We note that Compton heating or cooling by the quasar radiation itself has only a weak effect on the halo gas over a typical quasar lifetime. The characteristic timescale for Compton heating or cooling equals $\sim 3 \times 10^{8} L_{46}^{-1} r_{1}^{2}\left|1-2 T_{7} / \sigma_{200}^{2}\right|^{-1}$ yr, where $L_{46}$ is the quasar luminosity in units of $10^{46} \mathrm{ergs} \mathrm{s}^{-1}$, and $T_{7}$ is the Compton temperature of the quasar radiation in units of $10^{7} \mathrm{~K}$ (its typical value; see Mathews \& Ferland 1987). This timescale exceeds the Hubble time at $z \sim 3$ for $r \gtrsim 2 L_{46}^{1 / 2} \mathrm{kpc}$.

The surface brightness distribution of scattered light can be used to infer the density distribution of the halo gas. When combined with a measurement of the gas temperature, such data could constrain the mass profile of the dark matter as well. This method, however, might be compromised by quasar variability (Wise \& Sarazin 1992). The scattered light from a radius $r$ is delayed relative to the central quasar emission by $\sim 3 \times$ $10^{4}(r / 10 \mathrm{kpc}) \mathrm{yr}$, and so variability of the quasar on this timescale would affect the brightness distribution of the halo.

Detection of scattered light can also probe beaming in the quasar emission (Fabian 1989; Sarazin \& Wise 1993). In the case of beamed broad-line emission, the inferred electron tem-

\footnotetext{
${ }^{2}$ While it is possible also to search for broadening of stellar emission lines in starburst or normal galaxies, the scattered flux in these cases would typically be much lower than for bright quasars.
} 
perature would be uncertain by a factor of $(1-\cos \theta)^{-1}$ due to the unknown three-dimensional orientation of the beam relative to the line of sight. Also, the net polarization of the scattered light from the entire halo would not vanish in this case as it does in a spherical geometry. Nonsphericity or clumpiness in the distribution of infalling gas around the quasar host (Rees 1988) would result in similar effects.

In cases of beamed quasar emission, the contrast between the nuclear and host luminosities might be substantially reduced when the quasar beam is not directed at the observer. Indeed, the possibility that dust or electron scattering of quasar light accounts for the alignment between the radio and optical axes of radio galaxies is supported by observations of some objects (e.g., di Serego Alighieri et al. 1989; Dunlop \& Peacock 1993; Cimatti et al. 1993, 1994, 1997; Jannuzi et al. 1995; but see also Dey et al. 1997; Pentericci et al. 1998). However, only a small minority of all quasars are radio loud, and so these objects are by no means representative of the entire quasar population.

The existence of hot gas in halos of high-redshift galaxies without quasars can be probed by complementary measurements in the X-ray and radio bands. The forthcoming $A X A F^{3}$

${ }^{3}$ See http://asc.harvard.edu satellite will achieve arcsecond resolution and might detect free-free emission from the brightest halos. High-resolution radio telescopes, such as the VLA or MERLIN, could be used to search for the associated Sunyaev-Zeldovich effect of these halos and could provide an independent estimate of their temperature when combined with X-ray imaging data.

Finally, we note that the scattering efficiency of electrons or dust yields a maximum ratio between the luminosity of quasars and their hosts. Although the colors and morphology of the fuzz around low-redshift quasars indicate association with starlight (e.g., McLeod \& Rieke 1995; Bahcall et al. 1997), scattering may dominate the fuzz around high-redshift quasars at a time when galaxies have not yet converted most of their gas into stars. It would therefore be interesting to search for an extended scattering component with quasar colors in the photometry of high-redshift hosts (e.g., Kukula et al. 1998). The relative significance of starlight versus scattered quasar light would indicate whether stars preceded quasars throughout the entire history of galaxies.

This work was supported in part by the NASA grant NAG57039. I thank Daniel Eisenstein, Ari Laor, and George Rybicki for useful discussions.

\section{REFERENCES}

Adelberger, K. L., Steidel, C. C., Giavalisco, M., Dickinson, M., Pettini, M., \& Kellogg, M. 1998, ApJ, 505, 18

Bahcall, J. N., Kirhakos, S., Saxe, D. H., \& Schneider, D. P. 1997, ApJ, 479, 642

Bahcall, N. A., \& Chokshi, A. 1991, ApJ, 380, L9

Baugh, C. M., Cole, S., Frenk, C. S., \& Lacey, C. G. 1998, ApJ, 498, 504

Bregman, J. N., \& Glassgold, A. E. 1982, ApJ, 263, 564

Bremer, M. N., Fabian, A. C., Sargent, W. L. W., Steidel, C. C., Boksenberg, A., \& Johnstone, R. M. 1992, MNRAS, 258, 23P

Cassinelli, J. P., \& Hummer, D. G. 1971, MNRAS, 153, 9

Cimatti, A., Dey, A., van Breugel, W., Hurt, T., \& Antonucci, R. 1997, ApJ, 476, 677

Cimatti, A., di Serego Alighieri, S., Field, G. B., \& Fosbury, R. A. E. 1994, ApJ, 422, 562

Cimatti, A., di Serego Alighieri, S., Fosbury, R. A. E., Salvati, M., \& Taylor, D. 1993, MNRAS, 264, 421

Dey, A., \& Spinrad, H. 1996, ApJ, 459, 133

Dey, A., van Breugel, W., Vacca, W. D., \& Antonucci, R. 1997, ApJ, 490, 698

Dirac, P. A. M. 1925, MNRAS, 86, 825

di Serego Alighieri, S., Fosbury, R. A. E., Quinn, P. J., \& Tadhunter, C. N. 1989, Nature, 341, 307

Dunlop, J. S., \& Peacock, J. A. 1993, MNRAS, 263, 936

Ellingson, E., Green, R. F., \& Yee, H. K. C. 1991, ApJ, 378, 476

Fabbiano, G. 1989, ARA\&A, 27, 87

Fabian, A. 1989, MNRAS, 238, 41P

Fineschi, S., Kohl, J. L., Strachan, L., Gardner, L. D., Romoli, M., Huber, M. C. E., \& Noci, G. 1998, in The Corona and Solar Wind Near Minimum Activity, Fifth $\mathrm{SOHO}$ Workshop, ed. A. Wilson (ESA SP-404; Noordwijk: ESA), in press

Fisher, K. B., Bahcall, J. N., Kirhakos, S., \& Schneider, D. P. 1996, ApJ, 468, 469
Haehnelt, M. G., \& Rees, M. J. 1993, MNRAS, 263, 168

Haiman, Z., \& Loeb, A. 1998, ApJ, 503, 505

Hartwick, F. D. A., \& Schade, D. 1990, ARA\&A, 28, 437

Heckman, T. M., Lehnert, M. D., Miley, G., \& van Breugel, W. 1991a, ApJ, 381,373

Heckman, T. M., Lehnert, M. D., van Breugel, W., \& Miley, G. 1991b, ApJ, 370,78

Hu, E. M., McMahon, R. G., \& Egami, E. 1996, ApJ, 459, L53

Jannuzi, B. T., Elston, R., Schmidt, G. D., Smith, P. S., \& Atockman, H. S. 1995, ApJ, 454, L111

Kauffmann, G., White, S. D. M., \& Guiderdoni, B. 1993, MNRAS, 264, 201

Kukula, M. J., Dunlop, J. S., Hughes, D. H., Miller, L., O'Dea, C. P., \& Baum, S. A. 1998, NICMOS and the VLT: A New Era of High-Resolution NearInfrared Imaging and Spectroscopy, ed. W. Freudling \& R. Hook (ESO Conference and Workshop Proc. 55; Garching: ESO), 198

Magorrian, J., et al. 1998, AJ, 115, 2285

Mathews, W. G., \& Ferland, G. J. 1987, ApJ, 323, 456

McLeod, K. K., \& Rieke, G. H. 1995, ApJ, 454, L77

Mihalas, D. 1978, Stellar Atmospheres (2d ed.; San Francisco: W. H. Freeman), 420

Peterson, B. 1997, Active Galactic Nuclei (Cambridge: Cambridge Univ. Press), chap. 5

Pentericci, L., Rottgering, H. J. A., Miley, G. K., McCarthy, P., Spinrad, H., van Breugel, W. J. M., \& Macchetto, F. 1998, A \& A, in press (astro-ph/ 9809056)

Rees, M. J. 1988, MNRAS, 231, 91P

Rybicki, G. B., \& Hummer, D. G. 1994, A\&A, 290, 553

Sarazin, C. L., \& Wise, M. W. 1993, ApJ, 411, 55

Somerville, R. S., Primack, J. R., \& Faber, S. M. 1998, MNRAS, submitted (astro-ph/9806228)

Wise, M. W., \& Sarazin, C. L. 1992, ApJ, 395, 387 